\title{
Primary Industry of Employment
}

National Cancer Institute

\section{Source}

National Cancer Institute. Primary Industry of Employment. NCI Thesaurus. Code

C148246.

The main category of employment for an individual. 